\title{
A SULT2A1 genetic variant identified by GWAS as associated with low serum DHEAS does not impact on the actual DHEA/DHEAS ratio
}

\section{Robin Haring', Henri Wallaschofski', Alexander Teumer ${ }^{2}$, Heyo Kroemer ${ }^{3}$, Angela E Taylor ${ }^{4}$, Cedric H L Shackleton ${ }^{4}$, Matthias Nauck', Uwe Völker ${ }^{2}$, Georg Homuth ${ }^{2}$ and Wiebke Arlt ${ }^{4}$}

${ }^{1}$ Institute of Clinical Chemistry and Laboratory Medicine, ${ }^{2}$ Interfaculty Institute for Genetics and Functional Genomics, ${ }^{3}$ Department of Pharmacology, University Medicine Greifswald, Ferdinand-Sauerbruch Street, 17475 Greifswald, Germany

${ }^{4}$ School of Clinical and Experimental Medicine, Centre for Endocrinology, Diabetes and Metabolism, University of Birmingham, Birmingham, UK
Correspondence should be addressed to R Haring Email robin.haring@ uni-greifswald.de

\begin{abstract}
DHEA is the major precursor of human sex steroid synthesis and is inactivated via sulfonation to DHEAS. A previous genome-wide association study related the single nucleotide polymorphism (SNP) rs2637125, located near the coding region of DHEA sulfotransferase, SULT2A1, to serum DHEAS concentrations. However, the functional relevance of this SNP with regard to DHEA sulfonation is unknown. Using data from 3300 participants of the population-based cohort Study of Health in Pomerania, we identified 43 individuals being homozygote for the minor allele of the SNP rs2637125 (AA) and selected two sex- and agematched individuals with AG and GG genotype $(n=172)$ respectively. Steroid analysis including measurement of serum DHEA and DHEAS was carried out by liquid chromatography/mass spectrometry, employing steroid oxime analysis for enhancing the sensitivity of DHEA detection. We applied quantile regression models to compare median hormone levels across SULT2A1 genotypes. Median comparisons by SULT2A1 genotype (AA vs $A G$ and $G G$ genotypes respectively) showed no differences in the considered hormones including DHEAS, DHEA, androstenedione, as well as cortisol and cortisone concentrations. SULT2A1 genotype also had no effect on the DHEA/DHEAS ratio. Sex-stratified analyses, as well as alternative use of the SULT2A1 SNP rs182420, yielded similar negative results. Genetic variants of SULT2A1 do not appear to have an effect on individual DHEA and DHEAS concentrations or the DHEA/DHEAS ratio as a marker of DHEA sulfonation capacity.
\end{abstract}

\footnotetext{
Key Words

- DHEAS

- steroids

- genome-wide association study

- genetics

- epidemiology
}

Journal of Molecular Endocrinology (2013) 50, 73-77

\section{Introduction}

DHEAS is the most abundant steroid in human circulation and represents the conjugated, nonactive excretion form of DHEA, which acts as the universal sex steroid precursor and the major source of androgens in women
Published by Bioscientifica Ltd.

This is an Open Access article distributed under the terms of the Society for

Endocrinology's Re-use Licence which permits unrestricted non-commercial use 23 01:47:37AM distribution, and reproduction in any medium, provided the original work is properly cited. 
(Arlt et al. 1999). Serum DHEAS shows an age-related decline, and at 70 years of age, circulating DHEAS concentrations have diminished by $90 \%$ compared with the peak levels achieved at 20-30 years of age (Orentreich et al. 1984). This has led to widespread speculation about a causative role of DHEAS in the development of age-related diseases and longevity. A previous genome-wide association study (GWAS) comprising 14846 individuals identified eight common single nucleotide polymorphisms (SNPs) that implicate nearby genes as independently associated with serum DHEAS concentrations (Zhai et al. 2011), providing much needed insight into the metabolism of DHEAS. Among the identified genes, SULT2A1, a specialized sulfotransferase that converts DHEA to DHEAS in the adrenal cortex and liver, is an obvious candidate gene for subsequent functional studies (Rainey et al. 2002). Previous studies have established that the relationship between conjugated DHEAS and unconjugated DHEA is defined by SULT2A1 activity (Hammer et al. 2005) and that impairment of DHEA sulfonation results in low DHEAS and concurrent androgen excess with high DHEA and androstenedione levels (Noordam et al. 2009). But the clinical relevance of this candidate gene identified by GWAS with regard to individually meaningful prognostic, diagnostic, and therapeutic information is unknown. Therefore, we measured serum DHEAS, DHEA, and androstenedione concentrations in a subset of 215 individuals of the Study of Health in Pomerania (SHIP) follow-up cohort to investigate potential differences in adrenal androgens, including the DHEA/ DHEAS ratio, related to SULT2A1 genotypes.

\section{Materials and methods}

\section{Study population}

The SHIP is a population-based cohort study conducted in the northeastern area of Germany. Details on the SHIP study design, recruitment, and procedures have been published previously (Völzke et al. 2011). The net sample drawn for the SHIP comprised 6265 eligible individuals (3160 women) with German citizenship and main residency in the study area, whereof 4308 (2192 women) aged 20-80 years finally participated in the baseline study (SHIP-0) between 1997 and 2001 (response 68.8\%). During the 5-year follow-up (SHIP-1) between 2002 and 2006, 3300 (1711 women) participants were reexamined (response proportion 83.6\%). All participants gave informed written consent. A review board of independent scientists monitored the study. The study protocol is consistent with the principles of the Declaration of Helsinki, as reflected by an a priori approval of the Ethics Committee of the University of Greifswald. To assess the differences in adrenal androgens related to SULT2A1 genotype, we identified 43 individuals homozygote for the minor allele of SNP rs2637125 (AA), additionally selected two sex- and age-matched individuals having AG and GG genotype ( $n=86$ each) respectively, and finally measured adrenal androgens in the serum of these 215 participants of the follow-up study SHIP-1.

Anthropometric measurements were taken and BMI was calculated from the body weight in kilogram and height in meters according to the formula: $\mathrm{kg} / \mathrm{m}^{2}$. A summative cardiovascular disease (CVD) score was built upon diagnosis of angina pectoris, peripheral artery disease, heart failure, stroke, or myocardial infarction. HbA1c concentrations were determined by HPLC (Bio-Rad Diamat). Diabetes mellitus was defined based on selfreported physicians' diagnosis or use of antidiabetic medication (ATC code A10) in the last 7 days, or HbA1c concentrations $\geq 6.5 \%$. All blood samples had been drawn from the cubital vein between 0800 and $1800 \mathrm{~h}$ with participants in the supine position, and serum aliquots had been stored at $-80^{\circ} \mathrm{C}$ until analysis.

\section{Steroid analysis}

Steroids were measured by liquid chromatography/ tandem mass spectrometry (LC-MS/MS) employing a Waters Xevo mass spectrometer with Acquity uPLC system, fitted with a HSS T3, $1.8 \mu \mathrm{m}, 1.2 \times 50 \mathrm{~mm}$ column. LC-MS/MS conditions were an electrospray ionization source with capillary voltage $4.0 \mathrm{kV}$, a source temperature of $150{ }^{\circ} \mathrm{C}$, and a desolvation temperature of $500^{\circ} \mathrm{C}$. Steroid oxime analysis, which facilitates enhanced detection, i.e. increased sensitivity, by formation of oxime derivatives of the steroid oxo-groups (Kushnir et al. 2006), was employed for the measurement of DHEA and androstenedione and carried out in positive mode, whereas DHEAS measurement was performed in negative mode. DHEA, androstenedione, cortisol, and cortisone were extracted from $150 \mu \mathrm{l}$ serum via liquid-liquid extraction using $1 \mathrm{ml}$ tert-butyl-methyl-ether followed by derivatization into steroid oximes employing $100 \mu$ l derivatization mixture ( $0.16 \mathrm{~g}$ hydroxylamine in $8 \mathrm{ml}$ pyridine). For protein precipitation and extraction of DHEAS, $20 \mu \mathrm{l} \mathrm{ZnSO}_{4}$ $0.1 \mathrm{mM}$ and $100 \mu \mathrm{l}$ acetonitrile were added to $20 \mu \mathrm{l}$ serum before evaporation under constant flow of nitrogen. All steroids were separated using an optimized gradient system consisting of methanol with $0.1 \%$ formic acid and

Published by Bioscientifica Ltd. 
quantified referring to a linear calibration series with appropriate internal standards, ranging from 0.1 to $250 \mathrm{ng} / \mathrm{ml}$ for steroid oxime analysis and 250 to $100000 \mathrm{ng} / \mathrm{ml}$ for DHEAS analysis. Each steroid was identified by matching retention times and two mass transitions in comparison to a deuterated reference compound.

\section{Statistical analyses}

Continuous data are expressed as median (25th and 75th percentiles) and quantitative data as percentage. Given the broad age range of our study population, we performed linear regression analyses modeling continuous age and DHEA/DHEAS ratio variables, but without any indication of a potential age dependency. Individuals being homozygote for the rs2637125 minor allele (AA) were used as reference group (AG vs AA and GG vs AA). Alternatively, we also modeled the SULT2A1 SNP rs182420 with homozygous minor alleles (TT) as reference group (Goodarzi et al. 2007). Quantile regression models were used to compare median levels and calculate pairwise $P$ values for continuous variables. Differences in categorical variables across allele pattern were assessed by pairwise $\chi^{2}$ tests. To detect potential sex-specific differences, we repeated our analyses separately for men and women. All statistical analyses were performed using STATA 11.0 (Stata Corporation, College Station, TX, USA).

\section{Results}

Comparing median hormone levels by SULT2A1 genotype (AA vs AG and GG genotypes respectively), the conducted quantile regression models did not show any significant differences in the considered hormones including DHEA, DHEAS, cortisol, cortisone, or androstenedione concentrations (Table 1). SULT2A1 genotype also had no impact on the DHEA/DHEAS ratio. There were also no differences with regard to age, BMI, smoking, CVD, or diabetes mellitus. We found a borderline significant $(P$ value $=0.037)$ difference between AA and GG genotypes with regard to cortisol concentrations (Table 1). A scatterplot of the actual DHEA and DHEAS concentration by SULT2A1 genotype also failed to reveal clearly separated groups (Fig. 1). Analyses of the alternative SULT2A1 SNP rs182420 showed comparable negative results as for the main analysis, with similar borderline significant differences in cortisol concentrations ( $P$ value $=0.044)$ between TT and CC genotypes. Finally, sex-specific analyses of both SULT2A1 SNPs revealed a significantly higher DHEA/ DHEAS ratio among men with SULT2A1 SNP rs2637125 AA genotype compared with men with AG $(P$ value $=0.006)$ and GG $(P$ value $=0.019)$ genotype. As we identified no differences in blood sampling time between the groups, co-incidental group-specific variations in sampling time could be ruled out to explain this finding as a consequence of the diurnal rhythm of cortisol secretion.

\section{Discussion}

The present post-GWAS functional study revealed no significant differences in DHEA or DHEAS concentrations with regard to SULT2A1 genotype, a potential candidate gene predictive of low serum DHEAS concentrations

Table 1 Characteristics of the study population by SULT2A1 genotype (rs2637125). Values shown are expressed as median (25th and 75th percentiles) or percentage. The maximum number of observations was used for each measurement: DHEA: $n=214$, DHEAS: $n=204$, DHEA/DHEAS ratio: $n=204$, cortisol and cortisone: $n=213$. We used quantile regression models to compare median levels and calculate pairwise $P$ values for continuous variables and pairwise $\chi^{2}$ tests for categorical variables

\begin{tabular}{|c|c|c|c|c|c|}
\hline Variable & $\mathbf{A A}(n=43)$ & AG $(n=86)$ & $\begin{array}{l}\text { AG vs AA } \\
P \text { value }\end{array}$ & $\mathbf{G G}(n=86)$ & $\begin{array}{l}\text { GG vs AA } \\
P \text { value }\end{array}$ \\
\hline Age (years) & $49.0(40.0,72.0)$ & $50.0(43.0,72.0)$ & 0.867 & $49.5(42.0,72.0)$ & 0.989 \\
\hline DHEA (nmol/l) & $16.6(11.4,22.6)$ & $17.3(11.9,25.2)$ & 0.759 & $17.3(12.9,23.3)$ & 0.772 \\
\hline DHEAS (nmol/l) & $4767(2467,7841)$ & $5607(2337,10116)$ & 0.417 & $5735(3601,10689)$ & 0.320 \\
\hline DHEA/DHEAS ratio (nmol/I) & $0.004(0.002,0.005)$ & $0.003(0.002,0.006)$ & 0.506 & $0.003(0.002,0.005)$ & 0.446 \\
\hline Cortisol (nmol/l) & $81.9(51.1,122.9)$ & $89.8(64.4,142.5)$ & 0.593 & $108.7(65.7,143.9)$ & 0.037 \\
\hline Cortisone (nmol/l) & $31.8(23.6,44.7)$ & $38.9(30.0,53.1)$ & 0.189 & $41.1(29.2,52.5)$ & 0.071 \\
\hline Androstenedione (nmol/l) & $1.6(1.1,2.5)$ & $2.4(1.1,3.6)$ & 0.162 & $2.0(1.3,3.4)$ & 0.521 \\
\hline Blood sampling time ( $24 \mathrm{~h})$ & $11: 00(9,12)$ & $12: 00(10,13)$ & 0.450 & $11: 00(10,13)$ & 0.999 \\
\hline Current smoker (\%) & 37.2 & 30.2 & 0.173 & 30.2 & 0.379 \\
\hline BMI $\left(\mathrm{kg} / \mathrm{m}^{2}\right)$ & $26.9(24.5,29.8)$ & $26.7(24.7,29.3)$ & 0.815 & $27.9(25.1,32.2)$ & 0.269 \\
\hline Cardiovascular disease (\%) & 18.6 & 16.5 & 0.762 & 17.4 & 0.871 \\
\hline Diabetes mellitus (\%) & 11.6 & 9.3 & 0.679 & 9.3 & 0.679 \\
\hline
\end{tabular}

http://www.jme.endocrinology-journals.org DOI: $10.1530 / \mathrm{JME}-12-0185$ (c) 2013 Society for Endocrinology Printed in Great Britain
Published by Bioscientifica Ltd. 


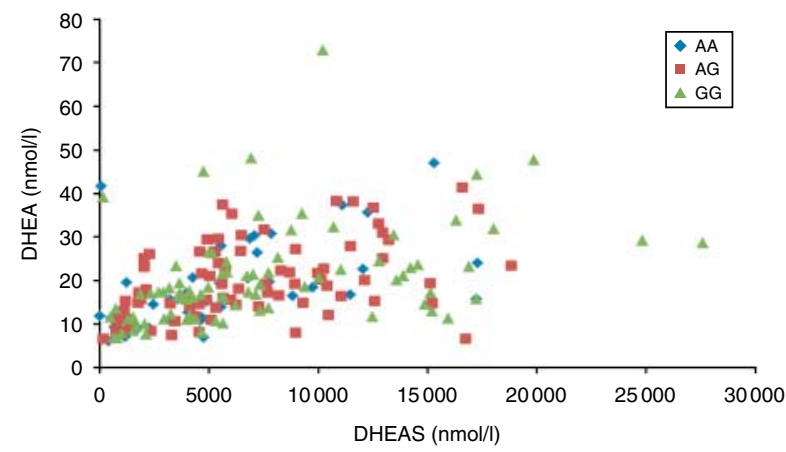

Figure 1

Concentrations of DHEA to DHEAS by SULT2A1 genotype. Individuals with AA genotype $(n=39)$ were compared with sex- and age-matched individuals carrying the AG genotype $(n=83)$ and GG genotype $(n=82)$ of the SNP rs2637125, all drawn from the SHIP follow-up study $(n=3300)$.

identified by a previous GWAS in 14846 Caucasian individuals (Zhai et al. 2011).

SULT2A1, also termed DHEA sulfotransferase, is a specialized sulfotransferase that catalyzes the sulfonation of steroids and detoxification of bile acids in the liver and adrenal glands (Strott 2002). In this study, we were not able to detect any differences in median DHEA and DHEAS concentrations across the different SULT2A1 genotype groups. Furthermore, SULT2A1 genotype showed no impact on the conversion of DHEA into DHEAS, as indicated by the lack of a significant difference between the group-specific DHEA/DHEAS ratio, previously established as a valid surrogate marker for DHEA sulfonation capacity (Arlt et al. 2006, Noordam et al. 2009). These findings are in line with previous studies lacking any impact of SULT2A1 allelic variants on serum adrenal hormones among AfricanAmerican men (Wilborn et al. 2006), Finnish prepubertal children (Utriainen et al. 2012), or white women with polycystic ovary syndrome (PCOS; Goodarzi et al. 2007). The latter study found only one particular SNP (rs182420) within the SULT2A1 gene related to lower DHEAS concentrations in women with PCOS, but not in women without PCOS (Goodarzi et al. 2007). Similarly, we were not able to detect an association of SULT2A1 SNP rs182420 with DHEA or DHEAS concentrations among men or women from the general population.

Although the applied SNP rs2637125 was not the most strongly $\left(P=2.61 \times 10^{-19}\right)$ associated with DHEAS concentrations in the previous GWAS (the strongest hit was rs11761528 on ZKSCAN5 with $P=3.15 \times 10^{-36}$ ), it was located only $12 \mathrm{~kb}$ downstream of the functionally very relevant protein-coding gene SULT2A1 (Zhai et al. 2011). However, potential explanations for the absent differences in the actual DHEA and DHEAS concentrations with regard to SULT2A1 genotype might relate to the unknown impact of this SNP on SULT2A1 transcription and expression. Previous studies examining regulatory mechanisms of SULT2A1 mRNA expression and enzymatic activity identified several factors as potent inhibitors of SULT2A1 expression (McAllister \& Hornsby 1988, Fang et al. 2007). For example, cytokines such as transforming growth factor- $\beta$ (TGF- $\beta$ ) and tumor necrosis factor- $\alpha$ (TNF- $\alpha$ ) have been found to decrease adrenal cell SULT2A1 mRNA levels (Parker et al. 1998). Decreased SULT2A1 activity was also observed during the acutephase response in a time- and dose-dependent manner in a murine model (Kim et al. 2004) and in patients with acute sepsis (Arlt et al. 2006). Also the transcriptional regulation via transcription factors SF-1 and GATA- 6 might be an important modulator of SULT2A1 expression and the production of adrenal DHEAS (Saner et al. 2005). In light of these limitations, the present data (although demonstrating no impact of SULT2A1 genotype on actual DHEA and DHEAS concentrations) could not ultimately rule out a potential influence of SULT2A1 on mechanisms regulating DHEAS production in the human adrenal.

Asking what GWAS will mean to the clinical endocrinologist, the present post-GWAS functional study puts further weight on the notion that candidate genes identified by GWAS do not necessarily translate into clinically meaningful prognostic, diagnostic, and therapeutic information for individuals (McCarthy 2009). However, given the generally small proportion of interindividual variability in disease predisposition explained by single genetic variants (Maher 2008), GWAS could only be a first step and essential starting point for the molecular classification of disease subtypes and the subsequent translation into novel therapeutic and preventative approaches (Haring \& Wallaschofski 2012).

\section{Declaration of interest}

The authors declare that there is no conflict of interest that could be perceived as prejudicing the impartiality of the research reported.

\section{Funding}

This work is part of the Community Medicine Research net (CMR) of the University Medicine Greifswald, Germany, which is funded by the Federal Ministry of Education and Research (grant no. ZZ9603), the Ministry of Cultural Affairs, as well as the Social Ministry of the Federal State of Mecklenburg - West Pomerania. The CMR encompasses several research projects that are sharing data of the population-based Study of Health in Pomerania (SHIP, http://www.medizin.uni-greifswald.de/icm/). This work is also part of the research project Greifswald Approach to Individualized

Published by Bioscientifica Ltd. 
Medicine (GANI_MED). The GANI_MED consortium is funded by the Federal Ministry of Education and Research and the Ministry of Cultural Affairs of the Federal State of Mecklenburg - West Pomerania (03IS2061A). This work was also supported by the Wellcome Trust (Project Grant 092283, to W A).

\section{References}

Arlt W, Callies F, van Vlijmen JC, Koehler I, Reincke M, Bidlingmaier M, Huebler D, Oettel M, Ernst M, Schulte HM et al. 1999 Dehydroepiandrosterone replacement in women with adrenal insufficiency. New England Journal of Medicine 341 1013-1020. (doi:10.1056/ NEJM199909303411401)

Arlt W, Hammer F, Sanning P, Butcher SK, Lord JM, Allolio B, Annane D \& Stewart PM 2006 Dissociation of serum dehydroepiandrosterone and dehydroepiandrosterone sulfate in septic shock. Journal of Clinical Endocrinology and Metabolism 91 2548-2554. (doi:10.1210/jc.20052258)

Fang HL, Strom SC, Ellis E, Duanmu Z, Fu J, Duniec-Dmuchowski Z, Falany CN, Falany JL, Kocarek TA \& Runge-Morris M 2007 Positive and negative regulation of human hepatic hydroxysteroid sulfotransferase (SULT2A1) gene transcription by rifampicin: roles of hepatocyte nuclear factor $4 \alpha$ and pregnane $\mathrm{X}$ receptor. Journal of Pharmacology and Experimental Therapeutics 323 586-598. (doi:10.1124/jpet.107.124610)

Goodarzi MO, Antoine HJ \& Azziz R 2007 Genes for enzymes regulating dehydroepiandrosterone sulfonation are associated with levels of dehydroepiandrosterone sulfate in polycystic ovary syndrome. Journal of Clinical Endocrinology and Metabolism 92 2659-2664. (doi:10.1210/jc.2006-2600)

Hammer F, Subtil S, Lux P, Maser-Gluth C, Stewart PM, Allolio B \& Arlt W 2005 No evidence for hepatic conversion of dehydroepiandrosterone (DHEA) sulfate to DHEA: in vivo and in vitro studies. Journal of Clinical Endocrinology and Metabolism 90 3600-3605. (doi:10.1210/jc. 2004-2386)

Haring R \& Wallaschofski H 2012 Diving through the "-omics": the case for deep phenotyping and systems epidemiology. OMICS 16 231-234. (doi:10.1089/omi.2011.0108)

Kim MS, Shigenaga J, Moser A, Grunfeld C \& Feingold KR 2004 Suppression of DHEA sulfotransferase (Sult2A1) during the acute-phase response. American Journal of Physiology. Endocrinology and Metabolism 287 E731-E738. (doi:10.1152/ajpendo.00130.2004)

Kushnir MM, Rockwood AL, Roberts WL, Pattison EG, Bunker AM, Fitzgerald RL \& Meikle AW 2006 Performance characteristics of a novel tandem mass spectrometry assay for serum testosterone. Clinical Chemistry 52 120-128. (doi:10.1373/clinchem.2005.052167)

Maher B 2008 Personal genomes: the case of the missing heritability. Nature 456 18-21. (doi:10.1038/456018a)
McAllister JM \& Hornsby PJ 1988 Dual regulation of 3ß-hydroxysteroid dehydrogenase, $17 \alpha$-hydroxylase, and dehydroepiandrosterone sulfotransferase by adenosine $3^{\prime}, 5^{\prime}$-monophosphate and activators of protein kinase $\mathrm{C}$ in cultured human adrenocortical cells. Endocrinology 122 2012-2018. (doi:10.1210/endo-122-5-2012)

McCarthy MI 2009 What will genome-wide association studies mean to the clinical endocrinologist? Journal of Clinical Endocrinology and Metabolism 94 2245-2246. (doi:10.1210/jc.2009-0403)

Noordam C, Dhir V, McNelis JC, Schlereth F, Hanley NA, Krone N, Smeitink JA, Smeets R, Sweep FC, Claahsen-van der Grinten HL et al. 2009 Inactivating PAPSS2 mutations in a patient with premature pubarche. New England Journal of Medicine 360 2310-2318. (doi:10.1056/NEJMoa0810489)

Orentreich N, Brind JL, Rizer RL \& Vogelman JH 1984 Age changes and sex differences in serum dehydroepiandrosterone sulfate concentrations throughout adulthood. Journal of Clinical Endocrinology and Metabolism 59 551-555. (doi:10.1210/jcem-59-3-551)

Parker CR Jr, Stankovic AK, Faye-Petersen O, Falany CN, Li H \& Jian M 1998 Effects of ACTH and cytokines on dehydroepiandrosterone sulfotransferase messenger RNA in human adrenal cells. Endocrine Research 24 669-673. (doi:10.3109/07435809809032668)

Rainey WE, Carr BR, Sasano H, Suzuki T \& Mason JI 2002 Dissecting human adrenal androgen production. Trends in Endocrinology and Metabolism 13 234-239. (doi:10.1016/S1043-2760(02)00609-4)

Saner KJ, Suzuki T, Sasano H, Pizzey J, Ho C, Strauss JF III, Carr BR \& Rainey WE 2005 Steroid sulfotransferase $2 \mathrm{~A} 1$ gene transcription is regulated by steroidogenic factor 1 and GATA-6 in the human adrenal. Molecular Endocrinology 19 184-197. (doi:10.1210/me.2003-0332)

Strott CA 2002 Sulfonation and molecular action. Endocrine Reviews 23 703-732. (doi:10.1210/er.2001-0040)

Utriainen P, Laakso S, Jaaskelainen J \& Voutilainen R 2012 Polymorphisms of POR, SULT2A1 and HSD11B1 in children with premature adrenarche. Metabolism 61 1215-1219. (doi:10.1016/j.metabol.2012. 02.008)

Völzke H, Alte D, Schmidt CO, Radke D, Lorbeer R, Friedrich N, Aumann N, Lau K, Piontek M, Born G et al. 2011 Cohort profile: the study of health in pomerania. International Journal of Epidemiology $\mathbf{4 0} 294-307$. (doi:10.1093/ije/dyp394)

Wilborn TW, Lang NP, Smith M, Meleth S \& Falany CN 2006 Association of SULT2A1 allelic variants with plasma adrenal androgens and prostate cancer in African American men. Journal of Steroid Biochemistry and Molecular Biology 99 209-214. (doi:10.1016/j.jsbmb.2006.01.006)

Zhai G, Teumer A, Stolk L, Perry JR, Vandenput L, Coviello A, Koster A, Bell D \& Wallaschofski H 2011 Eight common genetic variants associated with serum DHEAS levels suggest a key role in ageing mechanisms. PLoS Genetics 7 1-10. (doi:10.1371/journal.pgen. 1002025)

Received in final form 29 October 2012

Accepted 6 November 2012

Accepted Preprint published online 6 November 2012
(C) 2013 Society for Endocrinology Printed in Great Britain
Published by Bioscientifica Ltd 